\title{
An Iterative SVD-Tangential Interpolation Method for Medium-Scale MIMO Systems Approximation with Application on Flexible Aircraft
}

\author{
C. Poussot-Vassal
}

\begin{abstract}
In this paper, the problem of medium-scale MIMO LTI systems approximation is addressed. The proposed methodology, inspired from recent developments in the model reduction community (i.e. [1], [2], [3]), consists of combining the features of the SVD reduction approach with the tangential interpolation ones. The contributions of the paper are in two folds: $(i)$ it provides a simple but numerically robust and effective procedure, namely, Iterative SVD-Tangential Interpolation Algorithm (ISTIA), to approximate any medium-scale MIMO LTI system and, (ii) it assesses the efficiency of the proposed methodology on a medium-scale industrial aeroelastical aircraft model, on which different $\mathcal{H}_{\infty}$ control design methodologies are evaluated, illustrating on a complex industrial framework, the efficiency of the proposed approximation algorithm.
\end{abstract}

Index Terms-Model reduction, SVD, Tangential interpolation, Flexible aircraft, $\mathcal{H}_{\infty}$ control.

\section{INTRODUCTION}

\section{A. Motivations}

Computer-based dynamical system modeling softwares are now classically used by engineers to simulate, analyse, control and optimize a large variety of systems and phenomena with a high level of accuracy. The counterpart is that it leads to models with an increasing number of variables and resources to manage, resulting in an expensive numerical cost. Moreover, from the control engineer side, modern analysis and synthesis tools become drastically inefficient for such high dimensional dynamical systems (e.g. $\mu$-analysis, $\mathcal{H}_{\infty, 2}$ control... ). This is especially true in the flight dynamic domain where aircraft are modelled with high fidelity tools to account for the flexible modes and aeroelastic delays, which must be taken into consideration at the control design step. These observations, supported by the industrial partners demand for robust model reduction and control design methodologies, are the underlying justification for this work.

The main contributions of this paper are (i) to propose a new simple but effective mixed procedure gathering Singular Value Decomposition (SVD) and Tangential Interpolation techniques, called Iterative SVD-Tangential Interpolation Algorithm (ISTIA), to approximate any medium-scale Multiple Input Multiple Output (MIMO) Linear Time Invariant (LTI) system, and, (ii) to validate the proposed algorithm on a medium-scale industrial aeroelastical aircraft model, on

Author is with, Onera - The French Aerospace Lab, F-31055 Toulouse, France. http://www.onera.fr/staff-en/charles-poussot-vassal/

The research leading to these results has received funding from the European Union's Seventh Framework Program (FP7/2007-2013) for the Clean Sky Joint Technology Initiative under grant agreement $n^{\circ}$ CSJUGAM-SFWA-2008-001.

Author also acknowledge reviewer's very constructive feedbacks. which different $\mathcal{H}_{\infty}$-based control design methodologies are evaluated to achieve load alleviation and handling qualities.

\section{B. Paper structure \& Notations}

The rest of the paper is organized as follows: Section II reminds the MIMO LTI approximation problem and preliminary results. Section III presents the main contribution of the paper, namely, the ISTIA, with both implementation issues and performance comparison with classical techniques through usual model reduction benchmarks [4]. Section IV provides then a complete evaluation of this new technique on a complex industrial aeroelastic aircraft model plus a comparison of some of the recent $\mathcal{H}_{\infty}$ control design methods adjusted for load alleviation / handling quality purpose. Conclusion and discussion are gathered in Section V.

Mathematical notations are standard: the original system state is denoted $x \in \mathbb{R}^{n}$, the reduced-order system one is referred as $\hat{x} \in \mathbb{R}^{r}$. Full order MIMO LTI state-space (resp. transfer) form is denoted $\Sigma:=(A, B, C)$ (resp. $H(s):=$ $\left.C(s I-A)^{-1} B\right)$, and the reduced one $\hat{\Sigma}:=(\hat{A}, \hat{B}, \hat{C})$ (resp. $\left.\hat{H}(s):=\hat{C}(s I-\hat{A})^{-1} \hat{B}\right) . W$ and $V$ denote the left and right projectors, respectively. $V_{r}$ denotes the first $r$ columns of $V . \mathcal{V}=\operatorname{span}(v)$ means that the space $\mathcal{V}$ is spanned by the column vectors of $v$. Then, $\lambda($.$) holds for the$ eigenvalue operator, $\nabla($.$) stands for the Laplacian operator.$ The residual of the complex valued function $f(s)$ at $\lambda$ is denoted $\operatorname{res}(f(s), \lambda)=\lim _{s \rightarrow \lambda}(s-\lambda) f(s)$, where $s=j \omega$ (with $j=\sqrt{-1}$ ) defines the Laplace variable. The conjugate of $v$ is denoted $v^{*}$.

\section{MIMO LTI SYSTEMS APPROXIMATION PROBLEM}

\section{A. Preliminaries}

Let consider a stable and strictly proper MIMO LTI dynamical model $\Sigma:=(A, B, C)$ of order $n$ defined as:

$$
\Sigma:\left\{\begin{array}{l}
\dot{x}(t)=A x(t)+B u(t) \\
y(t)=C x(t)
\end{array}\right.
$$

where $A \in \mathbb{R}^{n \times n}, B \in \mathbb{R}^{n \times n_{u}}$ and $C \in \mathbb{R}^{n_{y} \times n}$. The projection-based approximation problem consists of finding $\hat{\Sigma}:=(\hat{A}, \hat{B}, \hat{C})$, a reduced-order model of order $r \ll n$, as

$$
\hat{\Sigma}:\left\{\begin{array}{l}
\dot{\hat{x}}(t)=\hat{A} \hat{x}(t)+\hat{B} u(t) \\
\hat{y}(t)=\hat{C} \hat{x}(t)
\end{array}\right.
$$

where $\hat{A}=W^{T} A V, \hat{B}=W^{T} B$ and $\hat{C}=C V$ (with $V, W \in$ $\mathbb{R}^{n \times r}, W^{T} V=I_{r}$ ) such that $\hat{\Sigma}$ well approximates $\Sigma$ in the sense of a given measure (evaluated here through a norm). Since the aim of model approximation is to capture the main system dynamics of interest and input/output behaviour, 
while guaranteeing stability and achieving minimal model mismatch, the $\mathcal{H}_{2}$ optimal approximation problem is often addressed. It consists of seeking an approximation $\hat{H}(s)$ of $H(s)$, such that,

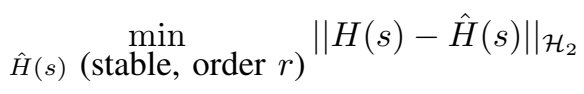

Moreover, in a control design perspective, attention to the $\mathcal{H}_{\infty}$ error should also be paid. Similarly, the $\mathcal{H}_{\infty}$ approximation problem thus consists of seeking $\hat{H}(s)$ such that,

$$
\min _{\hat{H}(s)(\text { stable, order } r)}\|H(s)-\hat{H}(s)\|_{\mathcal{H}_{\infty}}
$$

The latter problem (4) is practically very complex to achieve for large(medium)-scale models due to the (iterative) nature of the $\mathcal{H}_{\infty}$ norm computation, while, even if it is non-convex, first-order optimality conditions of the former one (3) can practically be characterized and satisfied (see [5], [6], [7], [3], [8], [9]). Let recall that the $\mathcal{H}_{2}$ norm of a $n$-th order strictly proper stable system $H(s)$ with simple poles at $\lambda_{1}$, $\lambda_{2}, \ldots, \lambda_{n} \in \mathbb{C}$ may be given as,

$$
\begin{aligned}
\|H(s)\|_{\mathcal{H}_{2}}^{2} & =\operatorname{trace}\left(B^{T} Q B\right)=\operatorname{trace}\left(C P C^{T}\right) \\
& =\sum_{i=1}^{n} \operatorname{res}\left(H^{T}(s), \lambda_{i}\right) H\left(-\lambda_{i}\right)
\end{aligned}
$$

where $Q$ and $P$ are the observability and controllability Gramian. Then, based on these formulations, the following theorems hold (due to [10], [1], [3]).

Theorem 1: Given $H(s):=C(s I-A)^{-1} B, \hat{H}(s):=$ $\hat{C}(s I-\hat{A})^{-1} \hat{B}$, the controllability and observability Gramian $\mathcal{P}_{e}=\left[\begin{array}{cc}P & X \\ X^{T} & \hat{P}\end{array}\right]$ and $\mathcal{Q}_{e}=\left[\begin{array}{cc}Q & Y \\ Y^{T} & \hat{Q}\end{array}\right]$, of $H(s)-$ $\hat{H}(s)$, if, $\hat{Q} \hat{P}+Y^{T} X=0, \hat{Q} \hat{B}+Y^{T} B=0$ and $\hat{C} \hat{P}-C X=$ 0 , then, with $\mathcal{J}=\|H(s)-\hat{H}(s)\|_{\mathcal{H}_{2}}$,

$$
\nabla_{\hat{A}} \mathcal{J}=0, \nabla_{\hat{B}} \mathcal{J}=0 \text { and } \nabla_{\hat{C}} \mathcal{J}=0,
$$

ensuring then the so-called Wilson first-order $\mathcal{H}_{2}$ conditions.

Based on Theorem 1, the reduced order model is obtained by linking projectors and stationary conditions as follows.

Theorem 2: At every stationary point of functional $\mathcal{J}$ (i.e. $\nabla \mathcal{J}=0$ ) where $\hat{P}$ and $\hat{Q}$ are invertible, we have the following identities: $\hat{A}=W^{T} A V, \hat{B}=W^{T} B$ and $\hat{C}=C V$ with $W^{T} V=I, W=-Y \hat{Q}^{-1}, V=X \hat{P}^{-1}$ and where $X$, $Y, \hat{P}$ and $\hat{Q}$ satisfies the Sylvester equations, related to the error system $H(s)-\hat{H}(s)$,

$$
\begin{array}{rlrl}
A P+P A^{T}+B B^{T} & =0 & Q A+A^{T} Q+C^{T} C & =0 \\
\hat{A} X^{T}+X^{T} A^{T}+\hat{B} B^{T} & =0 & A^{T} Y+Y \hat{A}-C^{T} \hat{C} & =0 \\
\hat{A} \hat{P}+\hat{P} \hat{A}^{T}+\hat{B} \hat{B}^{T} & =0 & \hat{Q} \hat{A}+\hat{A}^{T} \hat{Q}+\hat{C}^{T} \hat{C}=0
\end{array}
$$

Theorem 3: If $\nabla_{\hat{A}} \mathcal{J}=0, \nabla_{\hat{B}} \mathcal{J}=0$ and $\nabla_{\hat{C}} \mathcal{J}=0$, then the tangential interpolation conditions are satisfied for all $\hat{\lambda}_{i}$, $i=1, \ldots$, r, i.e.

$$
\begin{gathered}
{\left[H\left(-\hat{\lambda}_{i}\right)-\hat{H}\left(-\hat{\lambda}_{i}\right)\right] \hat{b}_{i}=0, \quad \hat{c}_{i}^{*}\left[H\left(-\hat{\lambda}_{i}\right)-\hat{H}\left(-\hat{\lambda}_{i}\right)\right]=0} \\
\left.\hat{c}_{i}^{*} \frac{d}{d s}[H(s)-\hat{H}(s)]\right|_{s=-\hat{\lambda}_{i}} \hat{b}_{i}=0
\end{gathered}
$$

where $\left\{\hat{b}_{1}, \ldots, \hat{b}_{r}\right\}=\hat{B}^{T} R$ and $\left\{\hat{c}_{1}, \ldots, \hat{c}_{r}\right\}=\hat{C} L$ (where $L$ and $R$ are the left and right eigenvectors associated to $\hat{\lambda}_{i}$, the eigenvalues of $\hat{A}$ ).

Then, [9] and [8] show that the Wilson conditions of Theorem 1 are equivalent to the tangential interpolation based optimality conditions of the Theorem 3 . Now, Theorem 4 shows how to construct the projectors $V$ and $W$ to fulfil any tangential interpolations [11], [3].

Theorem 4: Let $V \in \mathbb{C}^{n \times r}$ and $W \in \mathbb{C}^{n \times r}$ be matrices of full rank $r$ such that $W^{T} V=I_{r}$. Let $\sigma_{i} \in \mathbb{C}^{r}, \hat{b}_{i} \in \mathbb{C}^{n_{u}}$ and $\hat{c}_{i} \in \mathbb{C}^{n_{y}}$ (for $i=1, \ldots, r$ ) be given sets of interpolation points and left and right tangential directions, respectively. Assume that points $\sigma_{i}$ are selected such that $\sigma_{i} I_{n}-A$ are invertible. If, for $i=1, \ldots, r,\left(\sigma_{i} I_{n}-A\right)^{-1} B \hat{b}_{i} \in$ $\operatorname{span}(V)$ and $\left(\sigma_{i} I_{n}-A^{T}\right)^{-1} C^{T} \hat{c}_{i}^{*} \in \operatorname{span}(W)$, then, the reduced order system $\hat{H}$, satisfies the tangential interpolation conditions,

$$
\begin{gathered}
H\left(\sigma_{i}\right) \hat{b}_{i}=\hat{H}\left(\sigma_{i}\right) \hat{b}_{i}, \hat{c}_{i}^{*} H\left(\sigma_{i}\right)=\hat{c}_{i}^{*} \hat{H}\left(\sigma_{i}\right) \\
\hat{c}_{i}^{*} \frac{d}{d s} H\left(\sigma_{i}\right) \hat{b}_{i}=\hat{c}_{i}^{*} \frac{d}{d s} \hat{H}\left(\sigma_{i}\right) \hat{b}_{i}
\end{gathered}
$$

From Theorem 4, it turns that the $\mathcal{H}_{2}$ optimal problem consists of finding $\sigma_{i}, \hat{b}_{i}$ and $\hat{c}_{i}$ to satisfy Theorem 3 . This question is the underlying idea of many recent model reduction algorithms, aiming at finding the best interpolation points and direction. With reference to Theorems 1 and 2, similar research focus on the Gramian computations.

\section{B. Some literature results}

Methods allowing to reach these first-order optimality conditions have been widely explored and still are of great interest in both the numerical and control communities. Significant results in this field propose an iterative procedure allowing to converge toward a near optimal condition. The underlying idea is either to construct $X$ and $Y$ (when using the Sylvester-like approaches - Theorem 2) or to find the optimal interpolation points and directions (when using the Tangential approaches - Theorem 3). More specifically, from the Tangential(Krylov)-like side, the following techniques have retained our attention since they do exhibit very nice results in practice:

- The Iterative Rational Krylov Algorithm (IRKA), initially set for SISO systems [11] which shows excellent results on benchmarks but did not guarantee stability (unless implementing specific restart techniques). Later, in [12], authors extended it to MIMO systems, with a complex Trust Region algorithm, which guarantees convergence and preserves stability.

- At the same period, the Iterative Tangential Interpolation Algorithm (ITIA) for MIMO systems, suggested in [3], [8], was developed to handle the MIMO case. Indeed, the ITIA is similar to the MIMO IRKA. As the previous one, this procedure shows to be effective in many classical benchmark [4] but does not preserve stability, a priori. The contribution of [12] is to propose a guaranteed descent algorithm. 
In parallel to the Tangent(Krylov)-like approaches, from the Sylvester and SVD the sides, techniques have been developed to approximate MIMO LTI systems (without always aiming at guaranteeing $\mathcal{H}_{2}$ first-order optimality conditions), e.g.:

- The Balanced Truncation (BT), which is often considered as the gold standard since it preserves stability, provides a bound on the $\mathcal{H}_{\infty}$ error and a nearly optimal $\mathcal{H}_{2}$ one [13], [6]. The drawback is that it may practically fail when $n>500$.

- The Low Rank Square Root Method (LRSRM), which is a modification of the BT approach, is applicable for large-scale models but did not guarantee stability preservation [14].

- The Dominant Subspaces Projection Model Reduction (DSPMR), which is an heuristic approach allowing to handle large-scale systems. Unfortunately, this latter cannot guarantee stability neither [14].

- The Two-Sided Iterative Algorithm (TSIA) [9], which iteratively solves two Sylvester equations, is shown to be equivalent to the tangential interpolation. This procedure guarantees stability and provides nice results for medium-scale problems but suffers of two main drawbacks: first, it requires a good projector initialisation (i.e. $V$ and $W$ ) to converge, and secondly, no stopping criteria is described so far.

Many other methods exist, but the above ones catch our attention because of their presented efficiency. In the following section, grounded on [1], [3] and on [2] ideas, a simple but very effective hybrid approach is proposed.

\section{MAIN RESUlt: ItERATIVE SVD-TANGENTIAL INTERPOLATION ALGORITHM (ISTIA)}

\section{A. Main idea}

In [2], the author proposes an iterative SVD-Rational Krylov algorithm (ISRKA) to cope with instability problems of the Rational Krylov based techniques. Indeed, one projector is designed by solving one single Lyapunov function, guaranteeing system stability, while the second one is iteratively constructed through Rational Krylov techniques to achieve multi-shift moment matching, guaranteeing accurate (even if non $\mathcal{H}_{2}$-optimal) transfer matching (see also [15]). So far, the technique was appropriate for SISO, MISO and SIMO systems (for MIMO, deflation techniques were required). At almost the same period, in [3], authors propose a tangential interpolation approach to cope with the $\mathcal{H}_{2}$ firstorder optimality conditions for MIMO systems and suggest an iterative algorithm to find the interpolation points and directions. Here, the proposed idea consists of mixing these two approaches to approximate MIMO LTI systems by integrating both the SVD and the tangential interpolation approaches in order to (i) guarantee one side tangential interpolation at multiple shift points, iteratively selected to nearly reach the first-order $\mathcal{H}_{2}$ optimality conditions, and, (ii) guarantee the reduced system stability preservation at each step of the procedure thanks to the computation of one single Gramian. The proposed mixed approach, called
ISTIA, makes the solution easy to implement, provides an almost monotonic convergence and is adapted to SISO and MIMO LTI systems.

\section{B. ISTIA procedure}

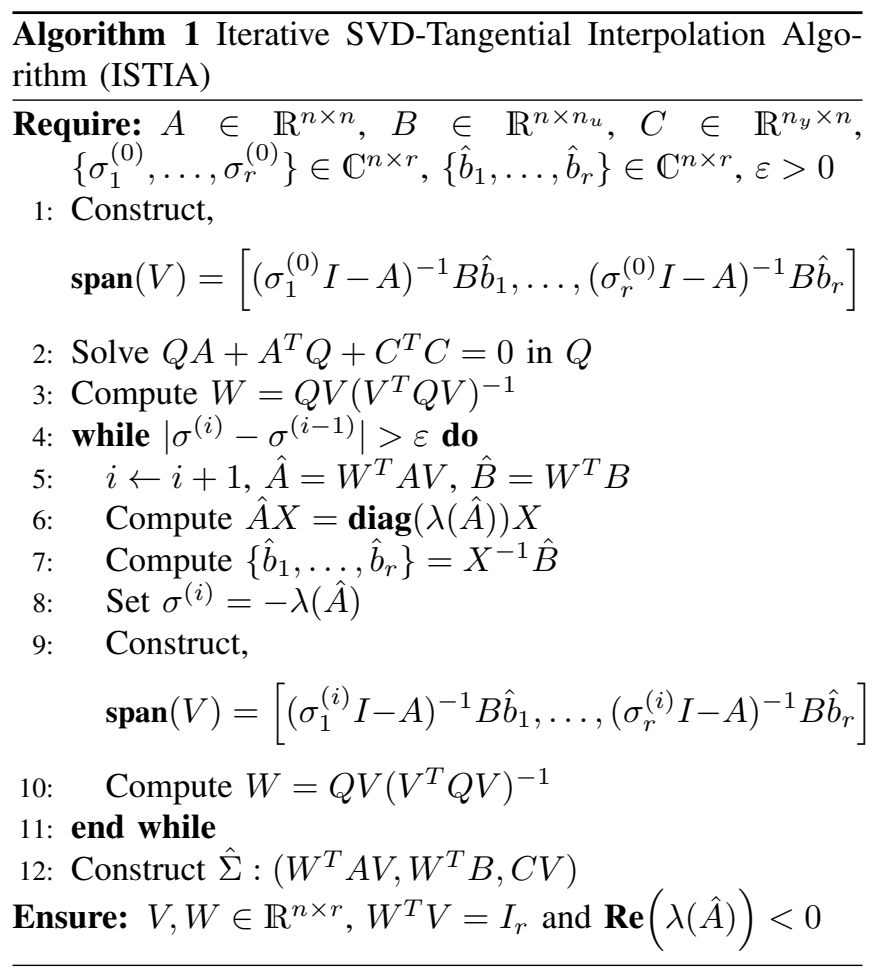

With reference to Algorithm 1, the procedure constructs the right projector $V$ thought tangential interpolation subspace at step 1 and computes one single Gramian (here the observability one) at step 2 . Then, for numerical purpose, the right projector $W$ is obtained by enforcing orthogonality, as in step 3 . Then, from step 4 to 11 , this procedure is repeated by using as new interpolation, points the mirror images of the eigenvalues of the reduced-order model, and, as new interpolation directions, the right eigenvectors associated with these eigenvalues (steps 6-8). The process is repeated until the interpolation points variation is smaller than a given tolerance $\varepsilon$.

\section{Algorithm properties and Implementation issues}

With reference to Algorithm 1, the following general remarks and implementation issues can be addressed:

- Since the Gramian is involved, system stability is preserved. The proof is easily obtained by considering, $Q=I_{n}$, thus $W=V$, which leads to $A^{T}+A+C^{T} C=$ 0 or equivalently (by left and right multiplying by $W$ and $V$ ), to $\hat{A}^{T}+\hat{A}+\hat{C}^{T} \hat{C}=0$.

- As the MIMO IRKA, the algorithm should converge toward optimal eigenvalues(vectors) and thus, ensure $\left(H\left(-\hat{\lambda}_{i}\right)-\hat{H}\left(-\hat{\lambda}_{i}\right)\right) \hat{b}_{i}=0$.

- Analogously, the controllability Gramian can be used instead of the observability one. In this case, the tangential subspace to be constructed is $\operatorname{span}(W)=$ 
$\left[\left(\sigma_{1}^{(i)} I-A^{T}\right)^{-1} C^{T} \hat{c}_{1}, \ldots,\left(\sigma_{r}^{(i)} I-A^{T}\right)^{-1} C^{T} \hat{c}_{r}\right]$ (where $\left\{\hat{c}_{1}, \ldots, \hat{c}_{r}\right\}=\hat{C} X$ ). In practice, this choice may be preferred when the observability Gramian is not satisfactory.

- Even if no proof can be established so far, the $\mathcal{H}_{\infty}$ system error remains quite reasonable as well. This property may be due to the Gramian computation.

- As in all Krylov-like procedures, to obtain real valued projection $V$ and $W$ matrices and increase computation speed, the starting shift grid should be either real or complex conjugate. Indeed, one can use the fact that, if, $v_{2}=v_{1}^{*}$, then $\operatorname{span}\left[v_{1}, v_{2}\right]=\operatorname{span}\left[\operatorname{Re}\left(v_{1}\right), \operatorname{Im}\left(v_{1}\right)\right]$.

- With reference to the ISTIA, experience shows that $\operatorname{span}(V)$ is much easily computed when the input/output magnitudes are of the same range. As a matter of fact, for implementation purpose, author proposes to pre-scale the $B$ and $C$ matrices.

- For computational improvements, it is possible to use low rank approximations of the $Q$ Gramian, but this case, stability is no longer guaranteed.

\section{Performance comparison on classical benchmark models}

To evaluate the proposed algorithm with respect to other literature methods, the ISTIA is applied on two benchmarks [4], namely, the SISO ISS-1R Module and the MIMO CD player. To evaluate the performances, the following relative $\mathcal{H}_{2}$ and $\mathcal{H}_{\infty}$ error metrics (in \%) will be used:

$$
\varepsilon_{\mathcal{H}_{2}}=100 \frac{\|\Sigma-\hat{\Sigma}\|_{\mathcal{H}_{2}}}{\|\Sigma\|_{\mathcal{H}_{2}}}, \varepsilon_{\mathcal{H}_{\infty}}=100 \frac{\|\Sigma-\hat{\Sigma}\|_{\mathcal{H}_{\infty}}}{\|\Sigma\|_{\mathcal{H}_{\infty}}}
$$

1) SISO subsystem of ISS-1R Module: To compare with respect to the complex but very powerful approach presented in [12] (namely MIMO IRKA or ITIA), the SISO subsystem of ISS-1R Module $(n=270)$ is considered. Table I compares the two approaches for $r=\{2,20\}$

\begin{tabular}{|c|c|c|}
\hline Method (with $r=2$ ) & $\varepsilon_{\mathcal{H}_{2}}[\%]$ & $\varepsilon_{\mathcal{H}_{\infty}}[\%]$ \\
\hline \hline [12], MIMO IRKA & 62.4 & N.A. \\
MIMO IRKA (our implementation) & $6.246 \times 10$ & $2.917 \times 10$ \\
Algorithm 1, ISTIA & $6.246 \times 10$ & $2.917 \times 10$ \\
\hline \hline Method (with $r=20$ ) & $\varepsilon_{\mathcal{H}_{2}}[\%]$ & $\varepsilon_{\mathcal{H}_{\infty}}[\%]$ \\
\hline [12], MIMO IRKA & $7.0 \times 10^{-1}$ & N.A. \\
MIMO IRKA (our implementation) & $5.034 \times 10^{-1}$ & $1.741 \times 10^{-1}$ \\
Algorithm 1, ISTIA & $5.034 \times 10^{-1}$ & $1.741 \times 10^{-1}$ \\
\hline
\end{tabular}

TABLE I

REDUCTION TECHNIQUES COMPARISON FOR THE ISS-1R MODULE.

With reference to Table I, it appears that our implementation of the MIMO IRKA (also denoted ITIA) is good. Indeed, for $r=2$, results are very similar, and, for $r=20$, even better than the one provided in [12]. Note that this is may be caused by the initial shift selection. Table I also illustrates that ISTIA, even if theoretically less optimal in the $\mathcal{H}_{2}$ sense, is very close to the optimal value (indeed, here it provides the same results as the ITIA). Since no illustration of MIMO cases are available in [12], next experiments will focus on a
MIMO benchmark without comparing with [12] but simply our implementation of the MIMO IRKA (denoted ITIA).

2) MIMO $C D$ player: The classical $\mathrm{CD}$ player model ( $n=120, n_{u}=2$ and $\left.n_{y}=2\right)$ is used here. For comparison, we apply the Balanced Truncation (BT, as implemented in Matlab), the ITIA and the proposed ISTIA techniques. Figure 1 gathers the relative $\varepsilon_{\mathcal{H}_{2}}$ and $\varepsilon_{\mathcal{H}_{\infty}}$ errors as a function of the approximation order $r$.
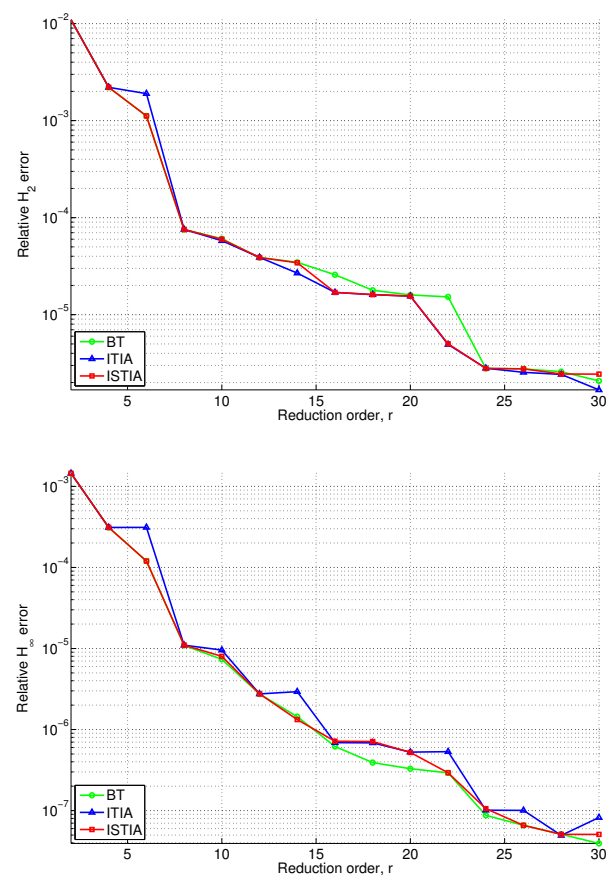

Fig. 1. $\varepsilon_{\mathcal{H}_{2}}$ (top) and $\varepsilon_{\mathcal{H}}$ (bottom) relative approximation errors as a function of the reduction order $r$ for the MIMO CD player system [4].

With reference to Figure 1, it is clear that the proposed ISTIA provides better $\varepsilon_{\mathcal{H}_{2}}$ results than the BT and is almost similar to the ITIA in most of the cases. Similarly, concerning the $\varepsilon_{\mathcal{H}_{\infty}}$ error, the ISTIA provides comparable performances with respect to the BT and is better than the ITIA. As a matter of fact, the ITIA seems to provide a very good $\varepsilon_{\mathcal{H}_{2}} / \varepsilon_{\mathcal{H}_{\infty}}$ error trade-off.

\section{ApPlication to AN INDUSTRial Flexible AIRCRAFT MODEL \& LOAD CONTROL DESIGN}

\section{A. Problem context}

Aircraft weight is a crucial design parameter for consumption and $\mathrm{CO}_{2}$ emission reduction. More specifically, wings weight dimensioning is a very complex task subject to a trade-off between Load Alleviation (LA) / Handling Qualities (HQ). Indeed, while load alleviation should be achieved to reduce the weight, load constraints level must be guaranteed in order to allow for accurately control the aircraft to fulfil the required flight quality level. Considering a flexible aircraft, the LA / HQ trade-off is even more complex to handle due to the closeness between flexible and rigid modes, and is thus a very challenging task in the aircraft industry. Moreover, 
today's available methods are not able to solve the robust $\mathcal{H}_{\infty}$ control design problem when the dimension of the system is too large. Indeed, synthesis is easier when model is low (i.e. $n<30$ ). It is why, here, we first apply the proposed reduction technique before synthesizing such a controller through different approaches (e.g. Riccati, LMIbased and non-smooth techniques), illustrating then the need for accurate and robust model approximation.

\section{B. Model description and approximation}

The considered model is an industrial longitudinal aeroelastic model with $n_{u}=2$ (the equivalent control surfaces and wind disturbance) and $n_{y}=4$ (the vertical load factor, the bending moment at the tail horizontal plan and wing/fuselage positions, and the pitch rate). This model also includes an actuator and a von-Karman wind disturbance model. It is worth being emphasized that approximating (and controlling) such system is a challenging task since the model order is about 300 , the conditioning number is very high and numerous badly damped mode are present. The ISTIA (Algorithm 1) approximation procedure is used on this industrial flexible aircraft model, and benchmarked with respect to the BT method. On Figure 2, the $\varepsilon_{\mathcal{H}_{2}}$ and $\varepsilon_{\mathcal{H}_{\infty}}$ errors are plotted as a function of the approximation order $r$.
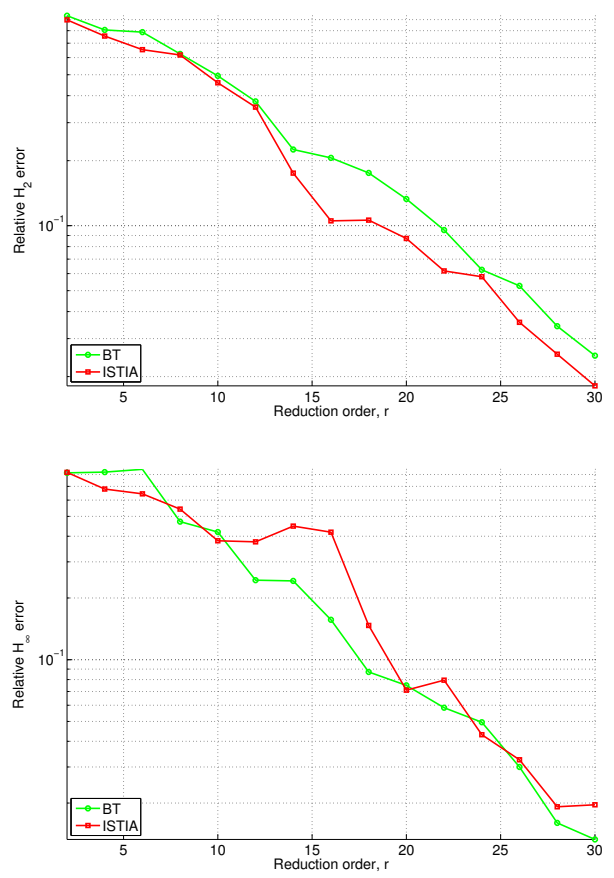

Fig. 2. $\varepsilon_{\mathcal{H}_{2}}$ (top) and $\varepsilon_{\mathcal{H}}$ (bottom) relative approximation errors as a function of the reduction order $r$ for the MIMO Aircraft system.

Figure 2 shows that the proposed algorithm outperforms the BT approach in term of error mismatch $\left(\varepsilon_{\mathcal{H}_{2}}\right)$ in all situations and keep the worst-case mismatch $\left(\varepsilon_{\mathcal{H}_{\infty}}\right)$ close to the BT one. Note that here, the ITIA is not presented due to stability issues encountered in many cases. Figure 3 compares the frequency responses and the pole locations

\begin{tabular}{|c|c|c|c|}
\hline Method (based on $\hat{\Sigma}$ with $r=16)$ & $\gamma_{\infty}$ & CPU [s] & $n_{c}$ \\
\hline \hline [16]: hinfsyn (Matlab, LMI) & 0.9022 & 2.91 & 20 \\
[17]: hinfsyn (Matlab, Riccati) & 0.9055 & 5.83 & 20 \\
[18]: YALMIP with SDP T3 [19], [20] & 1.2383 & 68.27 & 20 \\
[18]: YALMIP with SeDuMi [19], [21] & 1.2104 & 13.01 & 20 \\
[22]: hinfstruct (Matlab, 3 restarts) & 0.9026 & 3.93 & 20 \\
[22]: hinfstruct (Matlab, 3 restarts) & 0.9027 & 1.73 & 2 \\
\hline
\end{tabular}

TABLE II

SYNTHESIS METHODS COMPARISON.

between the original and reduced (obtained with the ISTIA, $r=16$ ) systems. With reference to theses figures, is clear that good fitting in term of frequency response and pole location are achieved. This last point is crucial for engineers, which are familiar with model modes physical meaning.

\section{Comparison of $\mathcal{H}_{\infty}$ control design methods}

Finally, to illustrate the interest of the proposed model reduction technique in a control framework, in this subsection we simply compare the closed-loop performances achieved with controllers adjusted with different $\mathcal{H}_{\infty}$ tuning techniques. The synthesis problem simply consists of (i) a load factor tracking and (ii) a wing/fuselage moment level attenuation. For synthesis, the aircraft load factor and wind/fuselage bending moments are considered to be measured, and the equivalent control surface is considered as the control signal. Table II summarizes the performances achieved by the different $\mathcal{H}_{\infty}$ minimization algorithms, where $n_{c}$ stands for the resulting controller order. Despite the difference in the results, all the different method show to successfully solve the considered problem (i.e. all controllers interconnected with the original system provide good closed-loop frequency responses). Nevertheless, the recently developed non-smooth based approach [22] still provides the best compromise between performance (i.e. $\gamma_{\infty}$ ), CPU time and resulting controller complexity. Figure 4 shows the frequency responses of the closed-loop including either the reduced or original system with the controller of order $n_{c}=2$, illustrating the fact that, by applying the controller synthesized on the reduced order model, still provides very satisfactory results on the original one (in term of the tracking and bending moment objectives).

\section{CONCLUSION}

In this paper the medium-scale MIMO LTI approximation problem is addressed. To handle practical problems, a simple but effective mixed algorithm, namely ISTIA, involving the SVD and the tangential interpolation techniques, is proposed. The method is positively validated on both classical model reduction benchmarks and on a complex industrial flexible aircraft on which control techniques are designed and validated (showing the consistency and the efficiency of the ISTIA in the control framework).

\section{REFERENCES}

[1] K. A. Gallivan, A. Vanderope, and P. Van-Dooren, "Model reduction of MIMO systems via tangential interpolation," SIAM Journal of Matrix Analysis and Application, vol. 26, no. 2, pp. 328-349, February 2004. 

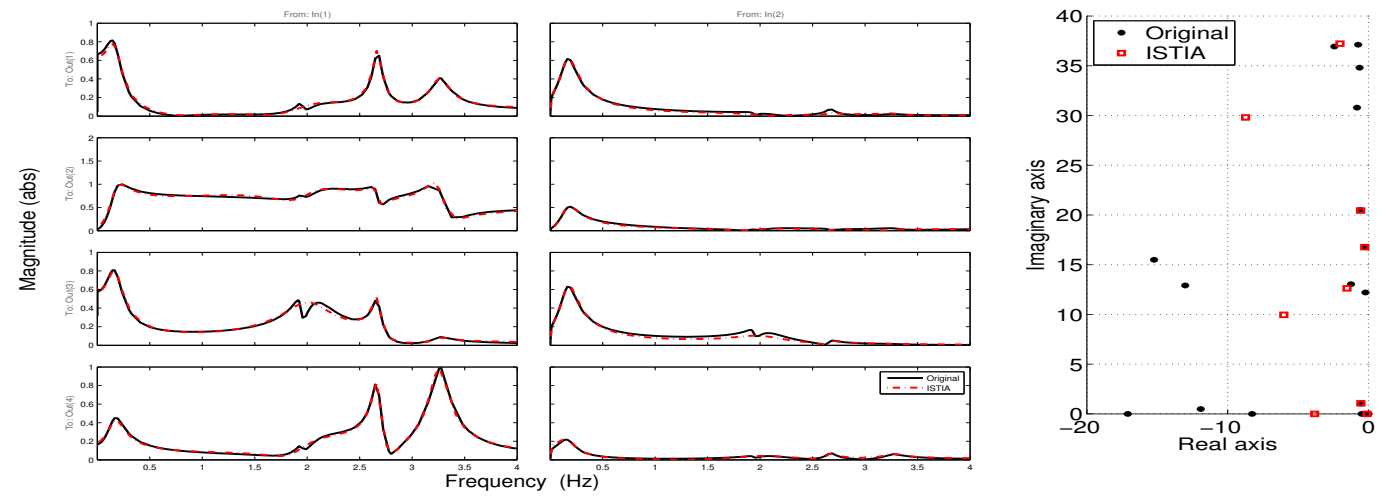

Fig. 3. Comparison of the Frequency responses (left) and Eigenvalues (right) between Original and Reduced models (obtained with ISTIA, $r=16$ ).

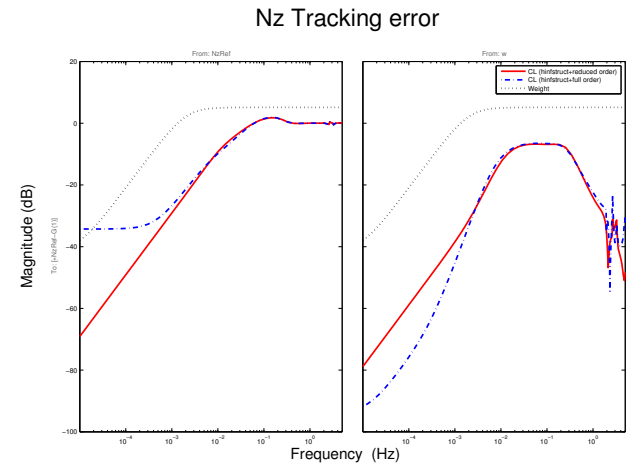

Wing/Fuselage $x$-axis bending moment

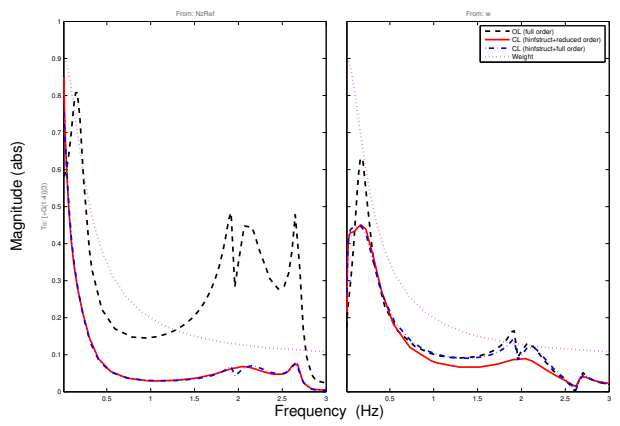

Fig. 4. Frequency responses obtained with an hinfstruct-based controller with $n_{c}=2$. Top: load factor tracking error in response to a set point (left) and vertical wind disturbance (right). Bottom: wing fuselage bending moment in response to a load factor set point (left) and vertical wind disturbance (right).

[2] S. Gugercin, "An iterative SVD-Krylov based method for model reduction of large-sale dynamical systems," Linear Algebra and its Applications, vol. 428, no. 8-9, pp. 1964-1986, April 2008.

[3] P. Van-Dooren, K. A. Gallivan, and P. A. Absil, " $\mathcal{H}_{2}$-optimal model reduction of MIMO systems," Applied Mathematics Letters, vol. 21, no. 12 , pp. 53-62, December 2008.

[4] Y. Chahlaoui and P. Van-Dooren, "A collection of benchmark examples for model reduction of linear time invariant dynamical systems; SLICOT Working Note," Florida State University and Université Catholique de Louvain, Tech. Rep., 2002.

[5] K. Zhou and J. C. Doyle, Essentials Of Robust Control. Prentice Hall, 1997.

[6] A. C. Antoulas, Approximation of Large-Scale Dynamical Systems.
Philadelphia: Advanced Design and Control, SIAM, 2005.

[7] S. Gugercin, A. C. Antoulas, and C. A. Beattie, "A Rational Krylov Iteration for Optimal $\mathcal{H}_{2}$ Model Reduction," in Proceedings of the 17th International Symposium on Mathematical Theory and Networks Systems, Kyoto, Japan, July 2006.

[8] P. Van-Dooren, K. A. Gallivan, and P. A. Absil, " $\mathcal{H}_{2}$-optimal model reduction with higher order poles," SIAM Journal on Matrix Analysis and Applications, vol. 31, no. 5, pp. 2738-2753, 2010.

[9] Y. Xu and T. Zeng, "Optimal $\mathcal{H}_{2}$ Model Reduction for Large Scale MIMO Systems via Tangential Interpolation," International Journal of Numerical Analysis, vol. 8, no. 1, pp. 174-188, 2010.

[10] D. A. Wilson, "Model reduction for multivariable systems," International Journal of Control, vol. 20, no. 1, pp. 57-64, 1974.

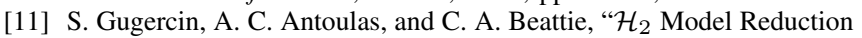
for Large Scale Linear Dynamical Systems," SIAM Journal on Matrix Analysis and Applications, vol. 30, no. 2, pp. 609-638, June 2008.

[12] C. Beattie and S. Gugercin, "A Trust Region Method for Optimal $\mathcal{H}_{2}$ Model Reduction," in Proceedings of the 48th IEEE Conference on Decision and Control, Shanghai, China, December 2009, pp. 53705375.

[13] K. Glover, "All Optimal Hankel Norm Approximation of Linear Multivariable Systems, and Their $\mathcal{L}_{\infty}$ error Bounds," International Journal Control, vol. 39, no. 6, pp. 1145-1193, 1984.

[14] T. Penzl, "LYAPACK A MATLAB Toolbox for Large Lyapunov and Riccati Equations, Model Reduction Problems, and Linear-Quadratic Optimal Control Problems Users' Guide (Version 1.0),” 2002.

[15] C. Poussot-Vassal and C. Roos, "Flexible Aircraft Reduced-Order LPV Model Generation from a Set of Large-Scale LTI Models," in Proceedings of the IEEE American Control Conference, San Francisco, California, USA, June 2011, pp. 745-750.

[16] P. Gahinet and P. Apkarian, "An linear matrix inequality approach to $\mathcal{H}_{\infty}$ control," International Journal of Robust and Nonlinear Control, vol. 4, no. 4, pp. 421-448, January 1994.

[17] J. C. Doyle, K. Glover, P. Khargonekar, and B. Francis, "State Space Solution to Standard $\mathcal{H}_{2}$ and $\mathcal{H}_{\infty}$ Control Problems," IEEE Transaction on Automatic Control, vol. 34, no. 8, pp. 831-847, December 1989.

[18] C. Scherer, P. Gahinet, and M. Chilali, "Multiobjective OutputFeedback Control via LMI Optimization," IEEE Transaction on Automatic Control, vol. 42, no. 7, pp. 896-911, July 1997.

[19] J. Lofberg, "YALMIP : A Toolbox for Modeling and Optimization in MATLAB," in Proceedings of the CACSD Conference, Taipei, Taiwan, 2004. [Online]. Available: http://control.ee.ethz.ch/ joloef/yalmip.php

[20] K. C. Toh, M. J. Todd, R. H. Tütüncü, and R. H. Tutuncu, "SDPT3 - a MATLAB software package for semidefinite programming," Optimization Methods and Software, vol. 11, pp. 545-581, 1999.

[21] J. F. Sturm, "Using SeDuMi 1.02, a MATLAB toolbox for optimization over symmetric cones," Optim. Methods Softw., vol. 11/12, no. 1-4, pp. 625-653, 1999, interior point methods.

[22] P. Apkarian and D. Noll, "Nonsmooth $\mathcal{H}_{\infty}$ Synthesis," IEEE Transaction on Automatic Control, vol. 51, no. 1, pp. 71-86, January 2006. 